\title{
Convergence study of isogeometric analysis based on Bézier extraction in electronic structure calculations
}

\author{
Robert Cimrman a,*, Matyáš Novák ${ }^{\mathrm{a}, \mathrm{b}}$, Radek Kolmanc, Miroslav Tưma ${ }^{\mathrm{d}}$, \\ Jiří Plešek ${ }^{c}$, Jiří Vackáŕ ${ }^{b}$ \\ ${ }^{a}$ New Technologies Research Centre, University of West Bohemia, Univerzitní 8, Plzeň 306 14, Czech Republic \\ b Institute of Physics, The Czech Academy of Sciences, Na Slovance 1999/2, Prague, Czech Republic \\ ${ }^{\mathrm{c}}$ Institute of Thermomechanics, The Czech Academy of Sciences, Dolejškova 5, Prague 182 00, Czech Republic \\ ${ }^{\mathrm{d}}$ Institute of Computer Science, The Czech Academy of Sciences, Pod Vodárenskou věži 2, 18207 Prague, Czech Republic
}

\section{A R T I C L E I N F O}

\section{Article history:}

Available online $\mathrm{xxx}$

PACS:

31.15.xv

03.65.Ge

02.70.Dh

\section{Keywords:}

Electronic structure calculation

Density functional theory

Finite element method

Isogeometric analysis

\begin{abstract}
A B S T R A C T
Behavior of various, even hypothetical, materials can be predicted via ab-initio electronic structure calculations providing all the necessary information: the total energy of the system and its derivatives. In case of non-periodic structures, the existing well-established methods for electronic structure calculations either use special bases, predetermining and limiting the shapes of wave functions, or require artificial computationally expensive arrangements, like large supercells. We developed a new method for non-periodic electronic structures based on the density functional theory, environment-reflecting pseudopotentials and the isogeometric analysis with Bézier extraction, ensuring continuity for all quantities up to the second derivative. The approach is especially suitable for calculating the total energy derivatives and for molecular-dynamics simulations. Its main assets are the universal basis with the excellent convergence control and the capability to calculate precisely the non-periodic structures even lacking in charge neutrality. Within the present paper, convergence study for isogeometric analysis vs. standard finite-element approach is carried out and illustrated on sub-problems that appear in our electronic structure calculations method: the Poisson problem, the generalized eigenvalue problem and the density functional theory Kohn-Sham equations applied to a benchmark problem.
\end{abstract}

(c) 2017 Elsevier Inc. All rights reserved.

\section{Introduction}

The electronic structure calculations represent a rigorous tool for predicting and understanding important properties of materials, such as elasticity, hardness, electric and magnetic properties, etc., see [34]. Those properties are tightly bound to the concept of the total internal energy of a system of atoms and its sensitivity with respect to various parameters, e.g., the atomic positions in order to reach a stable arrangement.

Computing the total internal energy of a system of atoms is a crucial task in electronic structure calculations. During the past decades, a whole range of ab-initio electronic structure calculation methods appeared in the fields of solid-state physics and material science, where, for example, behavior of dislocations, vacancies or other defects as well as interatomic potentials were studied. Electronic structure calculation methods can be employed to design new materials and structures such as thin-layered materials, nano-tubes or nano-wires. The existing well-established methods can be divided into two

\footnotetext{
* Corresponding author.

E-mail address: cimrman3@ntc.zcu.cz (R. Cimrman).
} 
distinctive groups: $k$-space methods based on the Bloch theorem [7,29] such as the plane-wave approach [1,28], and the real space methods with a special basis, such as the Gaussian basis [22]. This paper describes convergence aspects of our contribution to fill the gap among the existing methods via introducing a general, universal and in principle infinite basis within the framework of real-space methods, i.e. via creating a method that could be-within the real-space approach-a counterpart of what the plane-wave method is within the $k$-space approach.

In this paper, we study the convergence properties of the electronic structure calculation method presented in [13] based on

- the density functional theory (DFT), [21,34,38,41];

- the environment-reflecting pseudopotentials [50];

- a weak solution of the Kohn-Sham equations [30];

- isogeometric spatial discretization with the Bézier extraction [8].

The results are compared with the finite element discretization. The description of the essential characteristics of the real space code for electronic structure calculations can be found in [51]. Our electronic structure calculation method has been implemented in the open source finite element package SfePy [12] (Simple Finite Elements in Python, http://sfepy.org), which is a general package for solving (systems of) partial differential equations (PDEs) by the finite element method (FEM), cf. [45]. It has an interface to many external solvers and preconditioners, for example the UMFPACK sparse direct solver [18], PETSc iterative solvers and preconditioners [2], or pysparse eigenvalue solvers [24]. SfePy also supports distributed memory parallel computing with MPI using PETSc parallel vectors, matrices, and solvers as interfaced by the petsc4py package [17].

In recent past, SfePy has been extended with the isogeometric analysis (IGA) [14,15,27]-a spline-based modification of FEM. The key motivation for this extension, besides interesting convergence properties [32] in eigenvalue problems, was the possibility of a continuous field approximation with a high global continuity on a simple domain-a single NURBS (Non-uniform Rational B-spline) patch. This feature is crucial for an efficient evaluation of the sensitivity of the total energy with respect to atomic positions, also called the Hellman-Feynman forces (HFF), see [13], where continuous second derivatives of the discretized charge density and wave functions in the whole solution domain are prerequisites for practical HFF evaluation.

Recently, using FEM and its variants in electronic structure calculation context is pursued, see [20,35-37,40,46,49], including reduced order modeling, cf. [43], or adaptive partition of unity finite elements [19,39]. Other approaches to electronic structure calculations include the mesh-free approximation method [47], the coarse-graining method [48], or the quasicontinuum method [23].

In principle, IGA is a modification of FEM which employs shape functions with a higher order of continuity, such as B-splines, NURBS [42], T-splines [4], etc. It was successfully employed for numerical solutions of various physical and mathematical problems, such as fluid dynamics [3], diffusion and other problems of continuum mechanics [14,16,31,32]. The first application of IGA in electronic structure calculations has been published in [35].

It has been shown that the IGA produces optimal convergence rates and dispersion properties [26]. The IGA improves the dispersion errors and frequency spectrum in comparison with higher-order Lagrangian finite elements with $C^{0}$ continuity [26]. The convergence rate of IGA in linear free-vibration problems has been studied in [32]. The theoretical works relating to the convergence behavior of the IGA have been published in $[5,6,10]$, where effects of spline order and continuity degree have been also studied. These works also analyze how the accuracy of the IGA is influenced by the non-constant Jacobians of the mapping from the physical space to the parameter space. The convergence study of the IGA in two-dimensional Poisson problems for $C^{p-1}$ and $C^{0}$ continuities has been published in [16], where $p$ is a spline order. Convergence of the IGA with local refinement by subdivision was studied in [9].

We compare numerical convergence properties of FEM and IGA based on Bézier extraction using problems originating from various stages of the electronic structure calculation algorithm presented in [13], in order to assess the applicability of IGA for our purposes. The main contribution of the paper lies in assessing IGA convergence in the context of the new algorithm introduced in $[13,51]$-the algorithm results in a generalized eigenvalue problem where a rank-m update termarising from the separable form of pseudopotentials-is added to the sparse (Hamiltonian) matrix.

The paper is structured as follows: in Section 2 we provide a light-weight introduction to the topic of electronic structure calculations, in Section 3 the used discretization methods (FEM and IGA) are presented. Finally, in Section 4 the numerical convergence results are presented: first for several Poisson problems because the Poisson problem solution is an important part of the algorithm used for calculation of the electrostatic potential (see below); then several eigenvalue problems corresponding to simple quantum mechanical systems with a similar matrix structure to that of the complete problem; and finally, for the overall algorithm.

\section{Electronic structure calculations}

Let us briefly introduce the topic of electronic structure calculations adopted from [13]. The systems of atoms and molecules are described in the most general form by the many-particle Schrödinger equation, cf. [34],

$$
H \Psi\left(e_{1}, e_{2}, \ldots, e_{n}\right)=\varepsilon \Psi\left(e_{1}, e_{2}, \ldots, e_{n}\right),
$$

where $H$ is the Hamiltonian (energy operator) of the system, $e_{i}$ the particles (e.g. electrons) and $\varepsilon$ the energy of the state $\Psi$. Eq. (1) is, however, too complicated to solve, even for three electrons. Among the techniques reducing this complexity, 


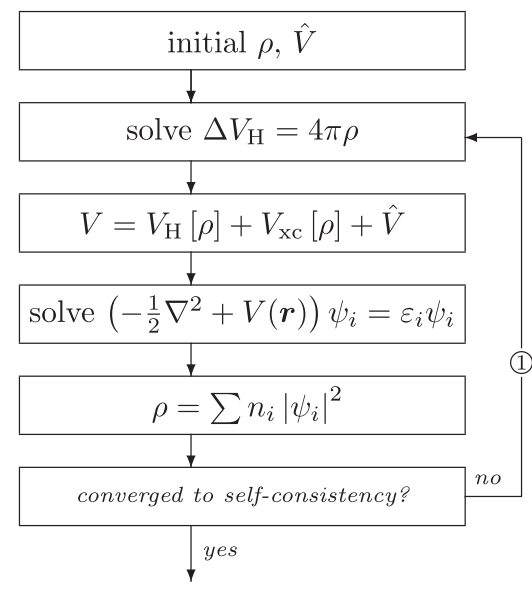

Fig. 1. DFT, iterative self-consistent scheme.

we use the DFT approach [21]. The DFT allows decomposing the many-particle Schrödinger equation into the one-electron Kohn-Sham equations [30]. Using atomic units they can be written in the common form

$$
\left(-\frac{1}{2} \nabla^{2}+V_{\mathrm{H}}(\boldsymbol{r})+V_{\mathrm{xc}}(\boldsymbol{r})+\hat{V}(\boldsymbol{r})\right) \psi_{i}=\varepsilon_{i} \psi_{i}
$$

and provide the orthonormal orbitals $\psi_{i}$ that reproduce, with the weights of occupations $n_{i}$, the charge density $\rho$ of the original interacting system, as

$$
\rho(\boldsymbol{r})=\sum_{i}^{N} n_{i}\left|\psi_{i}(\boldsymbol{r})\right|^{2} .
$$

$\hat{V}$ is a (generally) non-local Hermitian operator representing the effective ionic potential for electrons. In the present case, within the pseudopotential approach, $\hat{V}$ represents core electrons, separated from valence electrons, together with the nuclear charge. The separable form of pseudopotentials leads to a rank-m update term in the discretized form of $\hat{V}$ below and describes the external effect of the nucleus screened by the core (non-valence) electrons, as if that all were a single "blackbox-type" object, by means of the series of projection operators. The projectors forming the radial basis of the series are derived from corresponding radial wavefunctions (the series of mutually orthogonal wavefunctions with the same orbital angular momentum and varying number of nodes). $V_{\mathrm{xc}}$ is the exchange-correlation potential describing the non-Coulomb electron-electron interactions. The exact potential is not known, so we use the local-density approximation (LDA) of this potential [34], where the potential is a function of charge density at a given point. $V_{\mathrm{H}}$ is the electrostatic potential obtained as a solution to the Poisson equation. The Poisson equation for $V_{\mathrm{H}}$ has the charge density $\rho$ at its right-hand side and is as follows:

$$
\Delta V_{\mathrm{H}}=4 \pi \rho .
$$

Denoting the total potential $V:=V_{\mathrm{H}}+V_{\mathrm{xc}}+\hat{V}$, we can write, using Hartree atomic units,

$$
\left(-\frac{1}{2} \nabla^{2}+V(\boldsymbol{r})\right) \psi_{i}=\varepsilon_{i} \psi_{i}
$$

Note that the above mentioned eigenvalue problem is highly non-linear, as the potential $V$ depends on the orbitals $\psi_{i}$. Therefore an iterative scheme is needed, defining the DFT loop for attaining a self-consistent solution.

\subsection{DFT loop}

For the global convergence of the DFT iteration we use the standard algorithm outlined in Fig. 1. The purpose of the DFT loop is to find a self-consistent solution-a fixed point of a function of the charge density $\rho$. For this task, a variety of nonlinear solvers can be used. We use Broyden-type quasi-Newton solvers applied to

$$
\operatorname{DFT}\left(\rho^{i}\right)-\rho^{i}=\rho^{i+1}-\rho^{i}=0,
$$

where DFT denotes a single iteration of the DFT loop.

After the DFT loop convergence is achieved, the derived quantities, particularly the total energy, are computed. By minimizing the total energy as a function of atomic positions, the equilibrium atomic positions can be found. Therefore the DFT loop itself can be embedded into an outer optimization loop, where the objective function gradients are the HFF. 


\section{Discretization methods}

Before presenting key points of FEM and IGA, our problem needs to be reinstated in a weak form, usual in the finite element setting.

\subsection{Weak formulation}

Let us denote $H^{1}(\Omega)$ the usual Sobolev space of functions with $L^{2}$ integrable derivatives and $H_{0}^{1}(\Omega)=\left\{u \in H^{1}(\Omega) \mid u=\right.$ 0 on $\partial \Omega\}$.

The eigenvalue problem (5) can be rewritten using the weak formulation: find functions $\psi_{i} \in H^{1}(\Omega)$ such that for all $v \in H_{0}^{1}(\Omega)$ holds

$$
\int_{\Omega} \frac{1}{2} \nabla \psi_{i} \cdot \nabla v \mathrm{~d} V+\int_{\Omega} v V \psi_{i} \mathrm{~d} V=\varepsilon_{i} \int_{\Omega} v \psi_{i} \mathrm{~d} V
$$

The Poisson Eq. (4) has the following weak form:

$$
\int_{\Omega} \nabla v \cdot \nabla V_{\mathrm{H}}=4 \pi \int_{\Omega} \rho v
$$

Because of the finite domain, Dirichlet boundary condition need to be specified. We use zero boundary conditions for the eigenvalue problem (7) and the following boundary conditions for the Poisson Eq. (8):

$$
V_{\mathrm{H}}(\boldsymbol{r})=\int_{\Omega} \frac{\rho}{\left|\boldsymbol{r}-\boldsymbol{r}^{\prime}\right|} d \boldsymbol{r}^{\prime}, \boldsymbol{r} \in \partial \Omega
$$

Eqs. (7), (8) then need to be discretized-the infinite-dimensional functions are approximated by functions with a finite set of degrees of freedom (DOFs) and a basis, typically piece-wise polynomial:

$$
u(\boldsymbol{r}) \approx u^{h}(\boldsymbol{r})=\sum_{k=1}^{N} u_{k} \phi_{k}(\boldsymbol{r}) \text { for } \boldsymbol{r} \in \Omega,
$$

where $u$ is a continuous field ( $\psi, v, V_{H}$ in our equations), $u_{k}, k=1,2, \ldots, N$ are the discrete DOFs and $\phi_{k}$ are the basis functions. From the computational point of view it is desirable that the basis functions have a local support, so that the resulting system matrix is sparse.

Substituting (10) into (7) leads to the matrix form of the Kohn-Sham eigenvalue problem:

$$
\left(\boldsymbol{K}+\boldsymbol{V}\left(\psi_{i}\right)\right) \psi_{i}=\varepsilon_{i} \boldsymbol{M} \psi_{i},
$$

where

$$
\begin{aligned}
& \boldsymbol{K}=\left\{K_{i j}\right\} \equiv \int_{\Omega_{h}} \frac{1}{2} \nabla \phi_{i} \nabla \phi_{j}, \\
& \boldsymbol{V}\left(\psi_{i}\right)=\left\{V_{i j}\right\} \equiv \int_{\Omega_{h}} \phi_{i} V\left(\psi_{i}\right) \phi_{j}, \\
& \boldsymbol{M}=\left\{M_{i j}\right\} \equiv \int_{\Omega_{h}} \phi_{i} \phi_{j} .
\end{aligned}
$$

Similarly, the matrix form of the Poisson problem (8) is:

$$
\boldsymbol{K u}=\boldsymbol{f},
$$

where $V_{\mathrm{H}}(\boldsymbol{r}) \approx \sum_{k=1}^{N} u_{k} \phi_{k}(\boldsymbol{r})$ and $\boldsymbol{f}=\left\{f_{i}\right\} \equiv 2 \pi \int_{\Omega_{h}} \rho \phi_{i}$.

In our method $[13,51]$ the above matrices have the following properties after the discretization, independent whether IGA or FEM is used:

- $\boldsymbol{K}, \boldsymbol{M}$ are sparse matrices;

- $\boldsymbol{V}\left(\psi_{i}\right)$ has a sparse part and a dense part with rank-m update structure $\boldsymbol{U} \boldsymbol{C}_{\text {diag }} \boldsymbol{U}^{T}$, where $\boldsymbol{U}$ is a dense rectangular matrix with tens of columns per atom and $\boldsymbol{C}_{\text {diag }}$ is a diagonal matrix. The rank-m update part arises from the separable form of pseudopotentials.

\subsection{Finite element method}

In the FEM the discretization process involves the discretization of the domain $\Omega$-it is replaced by a polygonal domain $\Omega_{h}$ that is covered by small non-overlapping subdomains called elements (e.g. triangles or quadrilaterals in 2D, tetrahedrons or hexahedrons in 3D), cf. [25,45]. The elements form a finite element mesh.

The basis functions are defined as piece-wise polynomials over the individual elements, have a small support and are typically globally $C^{0}$ continuous. The discretized equations are evaluated over the elements to obtain local matrices or vectors that are then assembled into a global sparse system. The evaluation usually involves a numerical integration on a reference element, and a mapping to individual physical elements [25,45]. 


\subsection{Isogeometric analysis}

The basis functions in IGA are formed directly from the CAD geometrical description in terms of NURBS patches, without the intermediate FE mesh-the meshing step is removed, which is one of its principal advantages. A NURBS patch is a single NURBS object-a linear combination of control points $\boldsymbol{P}=\left\{\boldsymbol{P}_{A}\right\}_{A=1}^{N}$ (or unknown field coefficients) and NURBS basis functions $R_{A, p}(\underline{\xi})$, where $p$ is the NURBS solid degree and $\underline{\xi}=\left\{\xi_{1}, \ldots, \xi_{d}\right\}$ are the parametric coordinates. Thus, a d-dimensional geometric domain is defined by

$$
\boldsymbol{r}(\underline{\xi})=\sum_{A=1}^{n} \boldsymbol{P}_{A} R_{A, p}(\underline{\xi})=\boldsymbol{P}^{T} \boldsymbol{R}(\underline{\xi}) .
$$

If $d>1$, the NURBS solid can be defined as a tensor product of univariate NURBS curves. The basic properties of the B-spline basis functions can be found in [42].

The same NURBS basis is used also for the approximation of a continuous field $u\left(\psi, v, V_{H}\right.$ in our equations):

$$
\boldsymbol{u}(\underline{\xi})=\sum_{A=1}^{n} u_{A} R_{A, p}(\underline{\xi}),
$$

where $u_{A}$ are the unknown DOFs-coefficients of the basis in the linear combination.

Our implementation [11] uses a variant of IGA based on Bézier extraction operators [8] that is suitable for inclusion into existing FE codes. The code itself does not see the NURBS description at all. It is based on the observation that repeating a knot in the knot vector decreases continuity of the basis in that knot by one. This can be done in such a way that the overall shape remains the same, but the "elements" appear naturally as given by non-zero knot spans. The final basis restricted to each of the elements is formed by the Bernstein polynomials $\boldsymbol{B}$. The assembling of matrices and vectors resulting from (11) then proceeds in the usual FE sense (cf. [8]):

1. Setup points $\xi_{q}$ for the numerical quadrature on a reference element.

2. Loop over elements of the Bézier "mesh" (given by knot spans).

3. On each element $e$ :

(a) Evaluate the Bernstein basis $\left.\boldsymbol{B}\left(\xi_{q}\right)\right|_{e}$,

(b) Reconstruct the original NURBS basis: $\left.\boldsymbol{R}\left(\xi_{q}\right)\right|_{e}=\left.\left.\boldsymbol{C}\right|_{e} \boldsymbol{B}\left(\xi_{q}\right)\right|_{e}$, using the Bézier extraction operator $\boldsymbol{C}$, that is local to element $e$.

(c) Evaluate element contributions to the global matrix.

(d) Assemble using the original DOF connectivity.

The Bézier extraction matrices $\boldsymbol{C}$ are pre-computed for a NURBS patch domain using an efficient algorithm that employs the tensor-product nature of the patch [8], and then reused in all subsequent computations on that domain.

\section{Results}

The electronic structure calculations described in Section 2 involve solving the following two sub-problems:

- the Poisson Eq. (8) for the potential $V_{H}$,

- and the generalized eigenvalue problem (7).

Below we compare the convergence of FEM and IGA when applied to the two sub-problems, as well as to the entire algorithm of the DFT loop (6). All computations were performed on a tensor-product (cube) domain, with varying numbers $N_{e}$ of vertices/knots along the domain edge. The Lagrange polynomial basis was used in FEM, while the B-spline basis was applied in IGA-the NURBS basis was not needed because of the simple domain used.

We are interested in the convergence with respect to the following three parameters:

- The number of assembling cells (elements for FEM, knot spans/Bézier elements for IGA) $N_{c}$ provides insight into the work necessary to integrate over the domain.

- The number of non-zero entries in the matrices $N_{\mathrm{nz}}$ that corresponds to the cost of matrix-vector products, and hence the cost of a single linear/eigenvalue problem solver iteration.

- The total number of degrees of freedom $N_{\text {dof }}$, i.e. the size of the matrices, which is related to the difficulty, in terms of both time and memory requirements, of solving the Poisson Eq. (8) or the eigenvalue problem (7). Also this is the standard parameter for convergence studies.

Thus a higher $N_{c}$ indicates a higher cost of assembling the matrices, while higher $N_{\mathrm{nz}}$ and $N_{\text {dof }}$ mean a more difficult problem solution. Note that in this study we vary the above parameters by generating uniform meshes, see below. This is not optimal in terms of a desired accuracy, but serves well when comparing FEM and IGA. 

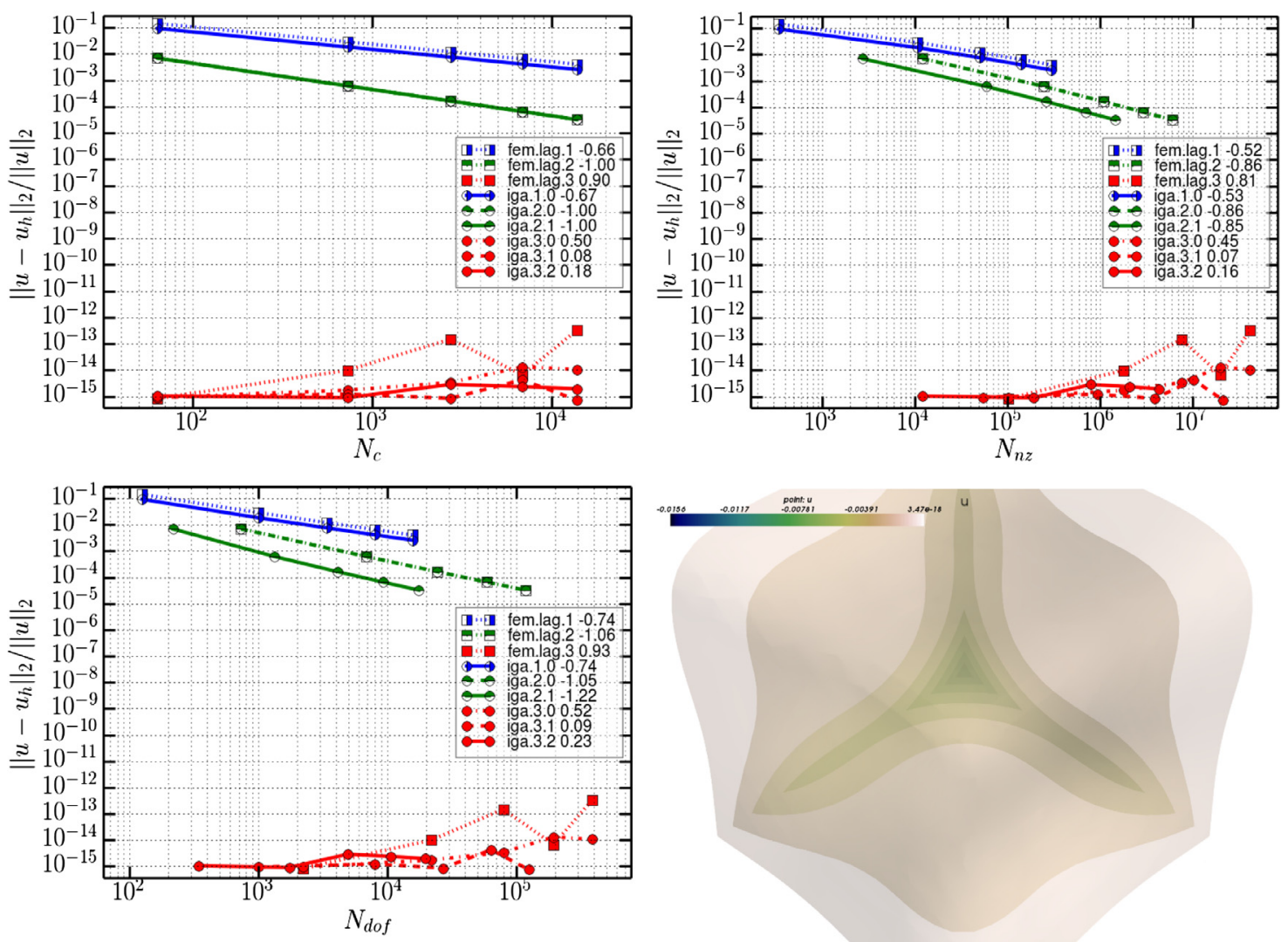

Fig. 2. Convergence results for $u(x, y, z) \equiv\left(x^{3}-0.125\right)\left(y^{3}-0.125\right)\left(z^{3}-0.125\right)$ with respect to the number of assembling cells $N_{c}$ (top-left), the number of non-zero entries in the matrices $N_{n z}$ (top-right) and the total number of degrees of freedom $N_{\text {dof }}$ (bottom-left). The legend for the FEM shows the polynomial order and the slope of the corresponding curve. For the IGA it shows the degree, the global continuity, and the slope, respectively. The isosurfaces of the function $u$ are shown in bottom-right.

\subsection{Poisson's equation with manufactured solutions}

In the method of manufactured solutions, cf. [33], a solution $u(\boldsymbol{r})$ is conceived and the left-hand side operator (the Laplace operator here) is applied to obtain the corresponding right-hand side $g(\boldsymbol{r}) \equiv \Delta u(\boldsymbol{r})$. The function $g(\boldsymbol{r})$ is then used as the right-hand side in the numerical solution, while $u(\boldsymbol{r})$ for $\boldsymbol{r} \in \Gamma$ is applied as the Dirichlet boundary condition on the whole domain surface $\Gamma$.

Several analytic formulas were considered in 3D. The domain was a unit cube $\Omega \equiv[-0.5,0.5]^{3}$, discretized using $N_{e}=5$, $10,15,20$ and 25 vertices/knots along the domain edge.

In the FEM setting, the tensor-product Lagrange basis with 1D polynomial orders 1, 2 and 3 and the uniform discretization were used. In the IGA context, the B-spline basis with 1D degrees 1, 2 and 3 and the approximation with uniformly distributed knots were used. We also varied the global continuity of the B-spline basis up to $C^{2}$, depending on the B-spline degree. Note that the B-spline basis with $C^{0}$ continuity is equivalent to the FEM basis.

The convergence of FEM and IGA for the Poisson problem for the two solutions considered (see below) is compared in Figs. 2, 3. The figures contain the convergence curves with respect to $N_{c}$ (top left), $N_{\text {nz }}$ (top right) and $N_{\text {dof }}$ (bottom left). The relative error $\left\|u-u_{h}\right\|_{2} /\|u\|_{2}$ is measured. The isosurfaces of the analytic solution $u$ are depicted in bottom-right. The figure legends use the following naming scheme:

- FEM Lagrange basis: "fem.lag. $<$ degree $><$ slope $>$ ",

- IGA basis: "iga. $<$ degree $>$. < continuity $><$ slope $>$ ",

where the "slope" is the slope of the line fitted by the least squares method to the corresponding convergence curve.

The conjugate gradient iterative solver from PETSc [2], preconditioned by the incomplete Cholesky decomposition was used for solving the linear system. The absolute precision for preconditioned residuals was set to $10^{-18}$, so that "exact" solutions were obtained.

The computations were done on a 4-CPU (8-core) Intel®Core ${ }^{\mathrm{TM}}$ i7-4770K CPU, $3.50 \mathrm{GHz}$, no parallelization was used. The matrix assembling times, linear system solving times and total elapsed times (wall time) in seconds for the largest grid size are shown in Table 1 . Increasing the continuity in IGA lead to a decrease in the solving time, while the assembling time 

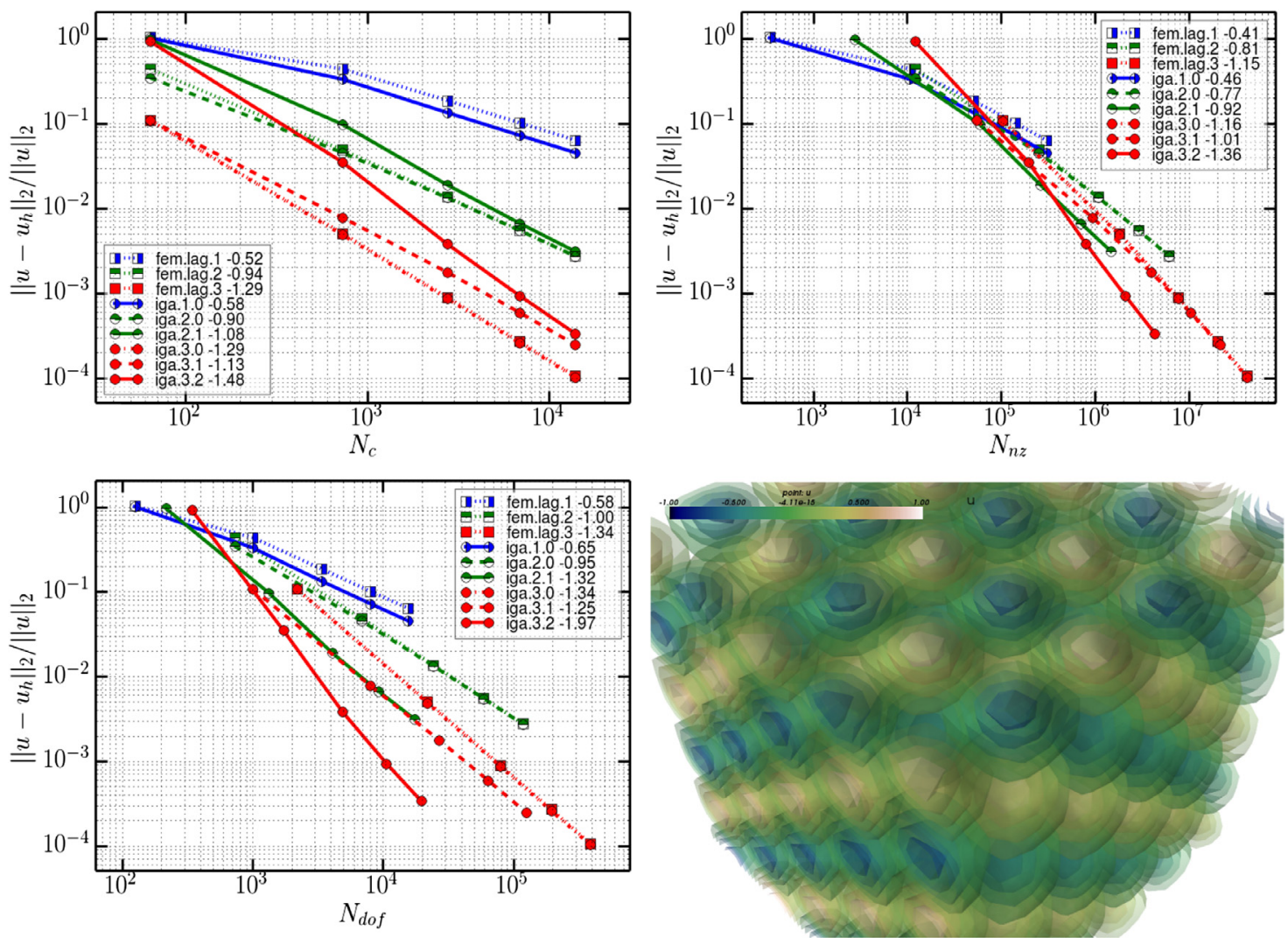

Fig. 3. Convergence results for $u(x, y, z) \equiv \sin (5 \pi x) \sin (5 \pi z) \cos (5 \pi y)$ with respect to the number of assembling cells $N_{c}$ (top-left), the number of non-zero entries in the matrices $N_{\mathrm{nz}}$ (top-right) and the total number of degrees of freedom $N_{\text {dof }}$ (bottom-left). The legend for the FEM shows the polynomial order and the slope of the corresponding curve. For the IGA it shows the degree, the global continuity, and the slope, respectively. The isosurfaces of the function $u$ are shown in bottom-right.

Table 1

Poisson problems: elapsed times for the largest FEM and IGA mesh sizes. The "polynomial" manufactured solution was $u(x, y, z) \equiv\left(x^{3}-0.125\right)\left(y^{3}-0.125\right)\left(z^{3}-0.125\right)$ and the "sine" solution was $u(x, y, z) \equiv \sin (5 \pi x) \sin (5 \pi z) \cos (5 \pi y)$. The total time denotes the wall time. All problems had the same number of assembling cells: $N_{c}=13,824$.

\begin{tabular}{lllllll}
\hline Solution & Basis & Continuity & $N_{\text {dof }}$ & Assembling $(\mathrm{s})$ & Solving $(\mathrm{s})$ & Total $(\mathrm{s})$ \\
\hline Polynomial & fem & 0 & 389,017 & 15.4 & 17.1 & 37.0 \\
Polynomial & iga & 0 & 389,017 & 15.0 & 7.5 & 35.1 \\
Polynomial & iga & 1 & 125,000 & 15.3 & 3.0 & 29.2 \\
Polynomial & iga & 2 & 19,683 & 16.7 & 0.4 & 29.1 \\
Sine & fem & 0 & 389,017 & 15.3 & 15.7 & 35.6 \\
Sine & iga & 0 & 389,017 & 14.6 & 7.0 & 31.9 \\
Sine & iga & 1 & 125,000 & 15.0 & 2.9 & 28.0 \\
Sine & iga & 2 & 19,683 & 16.0 & 0.4 & 28.2 \\
\hline
\end{tabular}

remained the same. This is consistent with the fact that all the corresponding grids had the same number of assembling cells.

\subsubsection{Polynomial tensor product solution}

The first solution $u(x, y, z) \equiv\left(x^{3}-0.125\right)\left(y^{3}-0.125\right)\left(z^{3}-0.125\right)$ (Fig. 2) is a tensor product of order three polynomials. The results reflect that as a numerical "zero" error is obtained independently of the resolution for the order/degree three bases. The higher continuity of IGA seems to mitigate a slight loss of precision due to round-off errors for higher resolutions.

\subsubsection{Sine tensor product solution}

The second solution $u(x, y, z) \equiv \sin (5 \pi x) \sin (5 \pi z) \cos (5 \pi y)$ (Fig. 3) was chosen so that it cannot be represented exactly in any basis. In terms of $N_{\text {dof }}$, increasing the global continuity of the IGA basis improves the convergence in comparison with 
R. Cimrman et al./Applied Mathematics and Computation 000 (2017) 1-15

Table 2

Simple eigenvalue problems: discretization parameters-numbers of vertices/knots along the domain edge and the domain edge length.

\begin{tabular}{lll}
\hline System & $N_{e}$ & Edge length $a$ \\
\hline 3D well & $5,10,20,35$ & 1 \\
3D oscillator & $5,10,20,35$ & 10 \\
2D hyperbolic & $20,50,100,250$ & 30 \\
\hline
\end{tabular}
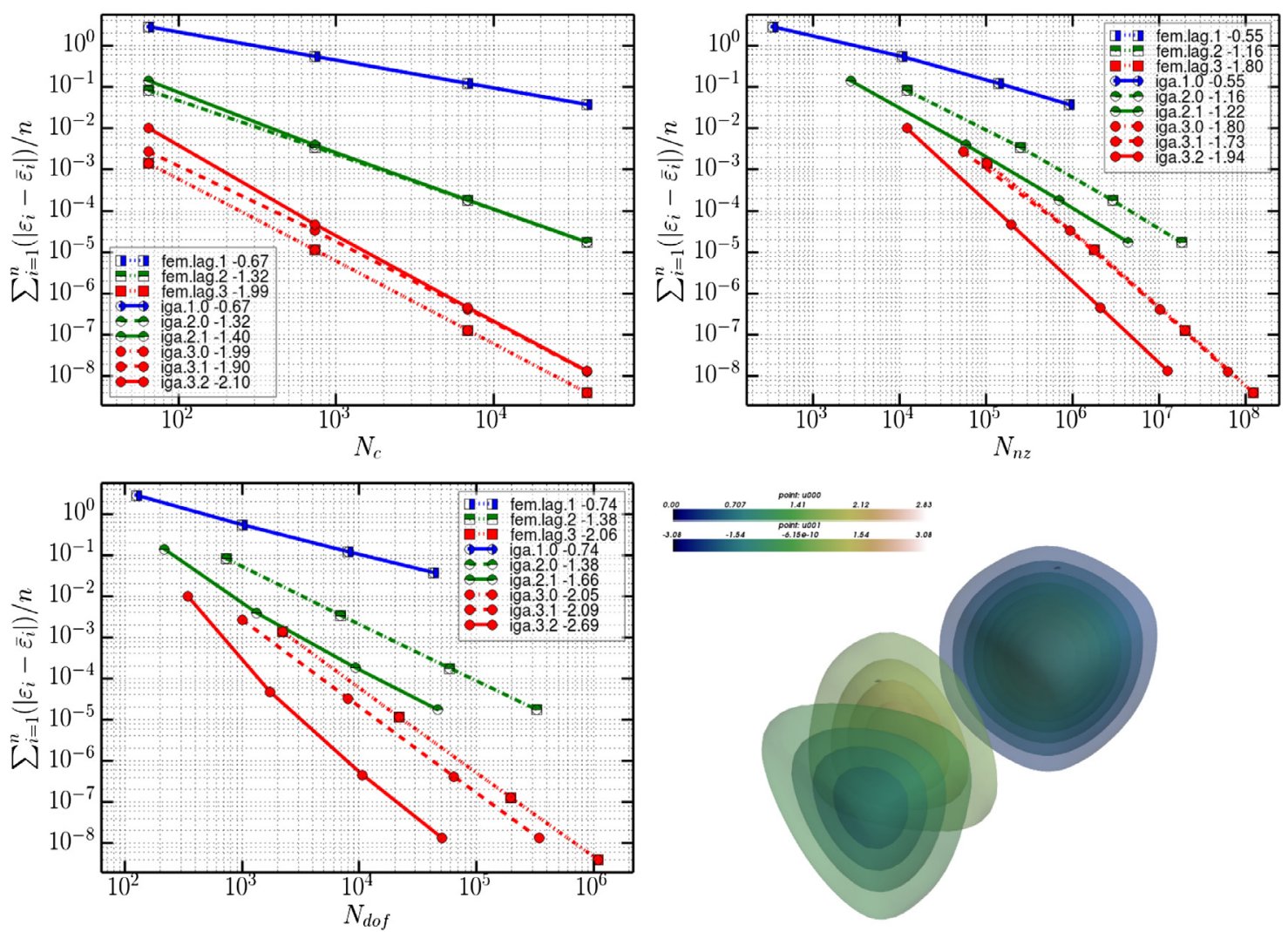

Fig. 4. Convergence results for the $3 \mathrm{D}$ well eigenvalue problem with respect to the number of assembling cells $N_{c}$ (top-left), the number of non-zero entries in the matrices $N_{\mathrm{nz}}$ (top-right) and the total number of degrees of freedom $N_{\mathrm{dof}}$ (bottom-left). The legend for the FEM shows the polynomial order and the slope of the corresponding curve. For the IGA it shows the degree, the global continuity, and the slope, respectively. The eigen-functions corresponding to the two smallest eigenvalues are shown in bottom-right.

FEM. The same holds for $N_{\mathrm{nz}}$ after a certain minimal system resolution. On the other hand, the standard $C^{0}$ basis performs better when considering the convergence with respect to $N_{c}$.

\subsection{Simple eigenvalue problems}

Several simple quantum mechanical systems were considered for our convergence study, namely an infinite potential well in 3D, a linear harmonic oscillator in 3D, and a hyperbolic 2D potential. The domain was a unit cube (or square): $\Omega \equiv[-a / 2, a / 2]^{d}, d=2,3$. The discretization parameters are summarized in Table 2 . We were interested in the convergence of the two smallest eigenvalues to the analytic values $\bar{\varepsilon}_{i}$. The error was measured using $\sum_{i=1,2}\left(\left|\varepsilon_{i}-\bar{\varepsilon}_{i}\right|\right) / 2$.

The convergence of FEM and IGA for the simple eigenvalue problems is compared in Figs. 4-6. All the figures contain the convergence curves with respect to $N_{c}$ (top left), $N_{\mathrm{nz}}$ (top right) and $N_{\text {dof }}$ (bottom left). The eigen-functions (isosurfaces in 3D) corresponding to $\varepsilon_{1}, \varepsilon_{2}$ are depicted in bottom-right. The figure legends use the same naming scheme as in Section 4.1. All eigenvalue problems in this section were solved using the JDSYM solver from Pysparse [24].

The computations were done on a 4-CPU (8-core) Intel ${ }^{\circledR}$ Core $^{\mathrm{TM}}$ i7-4770K CPU, 3.50 GHz, no parallelization was used. The matrix assembling times, eigenvalue problem solving times and total elapsed times (wall time) in seconds for the largest grid size are shown in Table 3. As in Section 4.1, increasing the continuity in IGA lead to a decrease in the solving time, while the assembling time remained the same for the grids with the same number of assembling cells. 

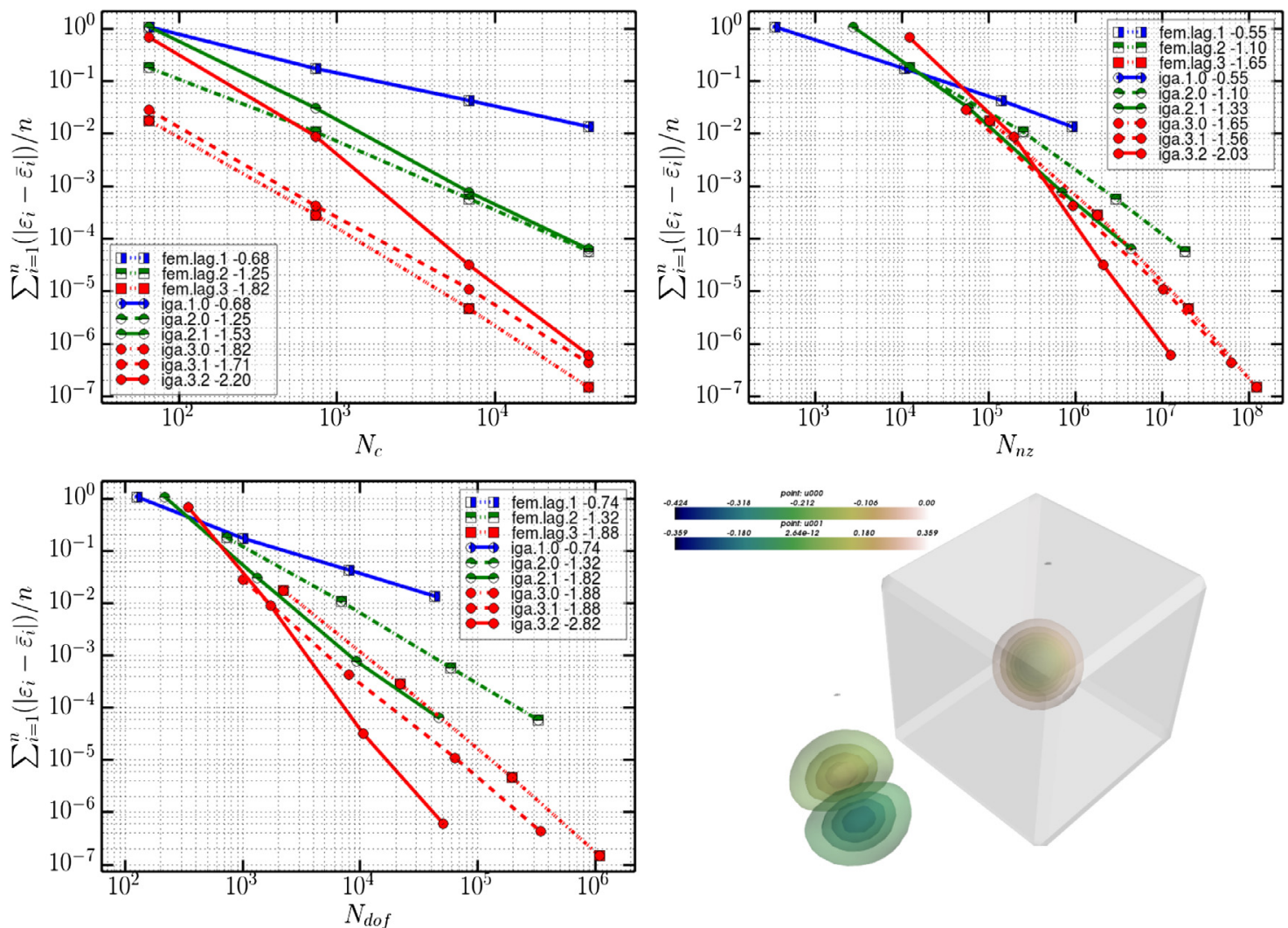

Fig. 5. Convergence results for the $3 \mathrm{D}$ oscillator eigenvalue problem with respect to the number of assembling cells $N_{c}$ (top-left), the number of nonzero entries in the matrices $N_{\mathrm{nz}}$ (top-right) and the total number of degrees of freedom $N_{\text {dof }}$ (bottom-left). The legend for the FEM shows the polynomial order and the slope of the corresponding curve. For the IGA it shows the degree, the global continuity, and the slope, respectively. The eigen-functions corresponding to the two smallest eigenvalues are shown in bottom-right.

Table 3

Simple eigenvalue problems: elapsed times for the largest FEM and IGA mesh sizes. The total time denotes the wall time. The number of assembling cells for 3D problems was $N_{c}=39,304$ and for 2D problems it was $N_{c}=62,001$.

\begin{tabular}{lllllrl}
\hline System & Basis & Continuity & $N_{\text {dof }}$ & Assembling $(\mathrm{s})$ & Solving $(\mathrm{s})$ & Total (s) \\
\hline 3D well & fem & 0 & $1,092,727$ & 127.0 & 327.5 & 463.3 \\
3D well & iga & 0 & $1,092,727$ & 135.2 & 196.9 & 348.0 \\
3D well & iga & 1 & 343,000 & 138.5 & 97.3 & 253.0 \\
3D well & iga & 2 & 50,653 & 145.8 & 20.8 & 189.1 \\
3D oscillator & fem & 0 & $1,092,727$ & 125.9 & 158.8 & 293.2 \\
3D oscillator & iga & 0 & $1,092,727$ & 131.7 & 185.0 & 332.7 \\
3D oscillator & iga & 1 & 343,000 & 137.0 & 90.9 & 245.3 \\
3D oscillator & iga & 2 & 50,653 & 146.2 & 21.4 & 190.6 \\
2D hyperbolic & fem & 0 & 559,504 & 3.2 & 135.4 & 139.1 \\
2D hyperbolic & iga & 0 & 559,504 & 3.7 & 59.0 & 63.7 \\
2D hyperbolic & iga & 1 & 250,000 & 3.8 & 22.1 & 26.8 \\
2D hyperbolic & iga & 2 & 63,504 & 4.0 & 3.9 & 9.0 \\
\hline
\end{tabular}

\subsubsection{Infinite potential well}

The 3D potential well can be described by (7) with a trivial choice of the potential: $V(\boldsymbol{r}) \equiv 0$. The exact eigenvalues are given by

$$
\bar{\varepsilon}_{i}=\frac{\pi^{2}}{2 a^{2}} c_{i}, c_{i}=3,6
$$

In our case, $\bar{\varepsilon}_{i}$ were equal to $14.804406601634037,29.608813203268074$.

The convergence curves are shown in Fig. 4. Here, IGA with the increasing continuity performs progressively better than FEM with the same polynomial order when the convergence with respect to $N_{\mathrm{nz}}$ and $N_{\text {dof }}$ is considered. On the other hand, its error is slightly higher than the FEM or $C^{0}$ IGA error for the $N_{c}$ curve. 

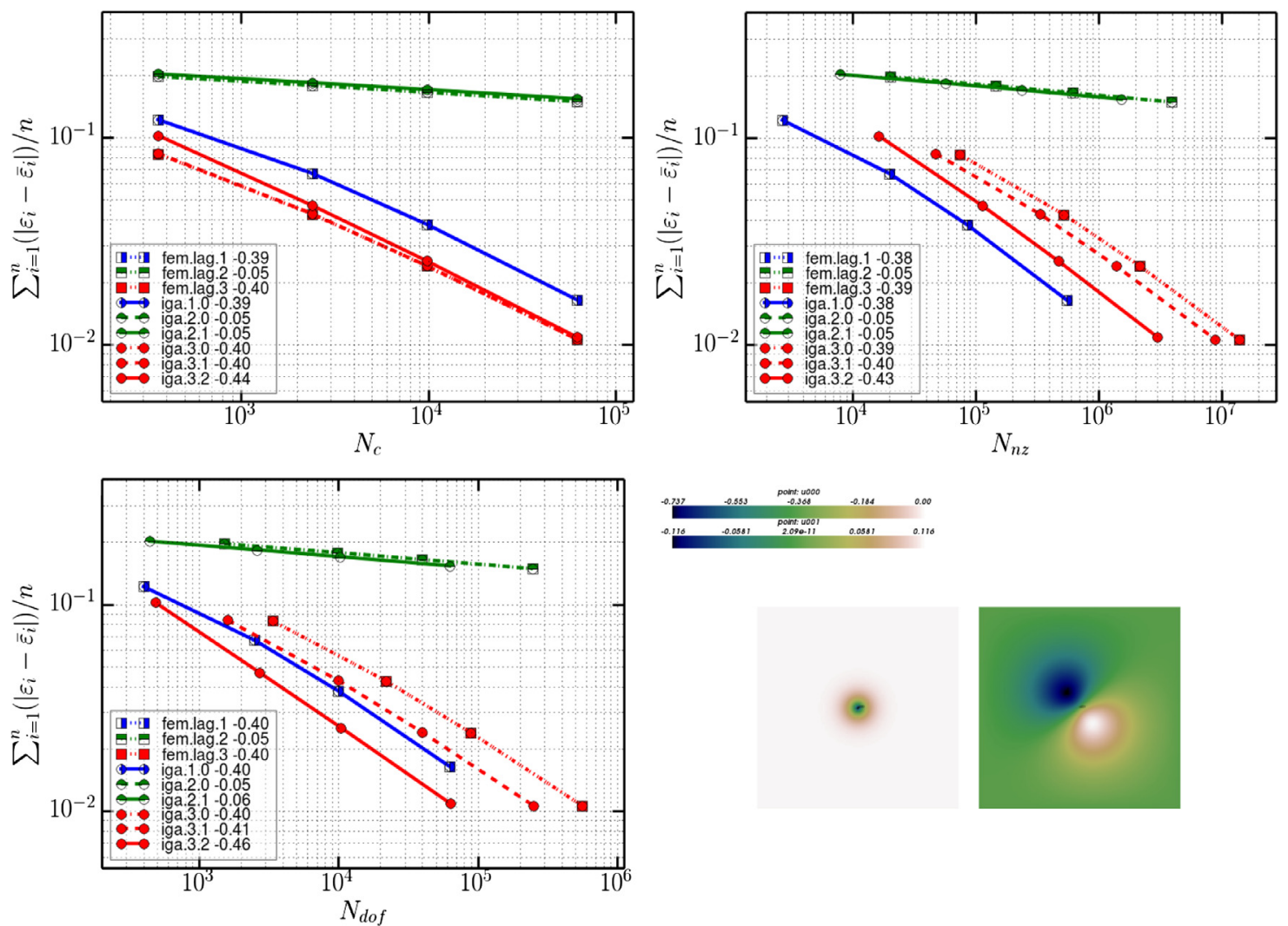

Fig. 6. Convergence results for the $2 \mathrm{D}$ hyperbolic eigenvalue problem with respect to the number of assembling cells $N_{c}$ (top-left), the number of nonzero entries in the matrices $N_{\mathrm{nz}}$ (top-right) and the total number of degrees of freedom $N_{\text {dof }}$ (bottom-left). The legend for the FEM shows the polynomial order and the slope of the corresponding curve. For the IGA it shows the degree, the global continuity, and the slope, respectively. The eigen-functions corresponding to the two smallest eigenvalues are shown in bottom-right.

\subsubsection{Linear harmonic oscillator}

The linear harmonic oscillator can be described by (7) with the following choice of the potential: $V(\boldsymbol{r}) \equiv \frac{1}{2} \boldsymbol{r}^{2}$. The exact eigenvalues $\bar{\varepsilon}_{i}$ are equal to $1.5,2.5$ in 3D.

The convergence curves are shown in Fig. 5 and qualitatively correspond to the 3D potential well results.

\subsubsection{Hyperbolic $2 D$ potential}

The hyperbolic 2D potential serves as a non-physical 2D analogy of Coulombic potential for hydrogen atom. It can be described by (7) with the following choice of the potential: $V(\boldsymbol{r}) \equiv-\frac{1}{2 \boldsymbol{r}}$. The exact eigenvalues $\bar{\varepsilon}_{i}$ are equal to $-0.5,-0.0 \overline{5}$, according to

$$
\bar{\varepsilon}_{i}=-\frac{1}{8(i-0.5)^{2}}, \text { where } i=1,2 .
$$

Unlike the previous examples, the potential $V(\boldsymbol{r})$ has a singularity at $\boldsymbol{r}=0$. To avoid numerical problems, the singularity was removed by replacing the values of radii smaller than a cut-off value $10^{-6}$ by this cut-off value. Due to that, the numerical solutions converged to slightly different values than those given above. Nevertheless, as can be seen in Fig. 6, the convergence of IGA solution with high global continuity seems to be better than that of FEM in terms of $N_{c}$ and $N_{\text {dof }}$, and worse in case of $N_{\mathrm{nz}}$.

Note that the singularity problem is not present in full DFT scheme below, thanks to the use of carefully chosen pseudopotentials.

\subsection{DFT loop}

The computations were performed on a cube domain having the edge size of 14 atomic units, with varying numbers $N_{e}$ of vertices/knots along its edge. A nitrogen atom was used for the benchmark due to availability of reference solution values for this simple system. The radial solution calculated under the same conditions (particularly the same pseudopotential and the same XC-term) was used as a reference solution. In our computations there are four occupied states with two distinct energy levels (single + triple eigenvalues, $\varepsilon_{1}, \varepsilon_{2}$ respectively)-the core energy level (1s) is hidden in the pseudopotential and 

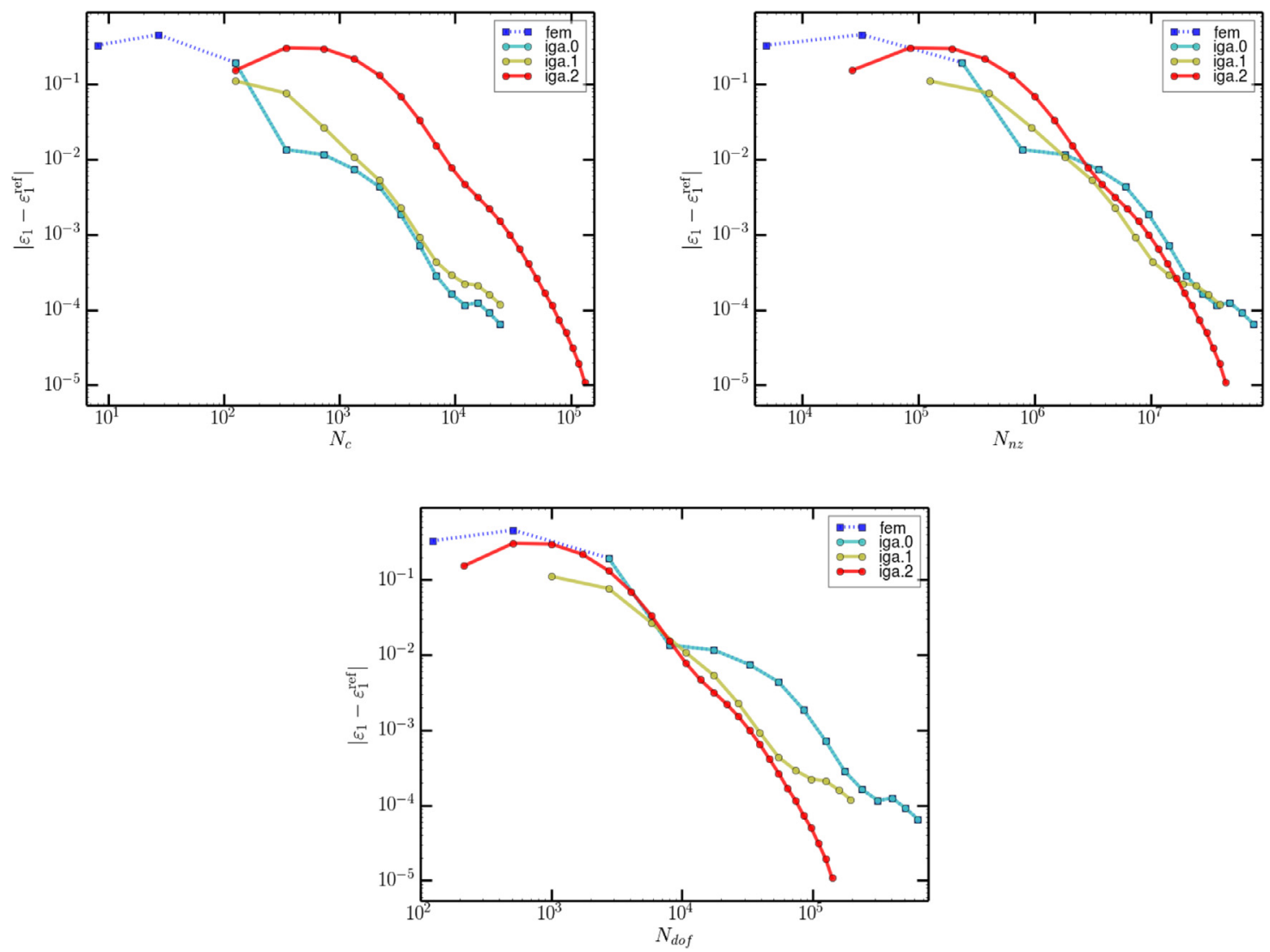

Fig. 7. Convergence with respect to the number of assembling cells $N_{c}$ (top-left), the number of non-zero entries in the matrices $N_{\mathrm{nz}}$ (top-right) and the total number of degrees of freedom $N_{\text {dof }}$ (bottom) of the first eigenvalue $\varepsilon_{1}$ : difference with respect to a reference value. The IGA labels indicate the global basis continuity. The tri-cubic FEM basis and degree three IGA (B-spline) basis were used.

thus not explicitly included in this DFT loop calculation. The tri-cubic FEM (Lagrange) basis and degree three IGA (B-spline) basis were used. The continuities $C^{0}, C^{1}$ and $C^{2}$ were used for the IGA basis.

In Figs. 7and 8 we can see the convergence of the complete DFT loop, involving repeated iterative solution of the Poisson problem (12) and the Kohn-Sham problem (11), to reference values: $\left|\varepsilon_{i}-\varepsilon_{i}^{\text {ref }}\right|, i=1,2$ is measured. Fig. 9 depicts the convergence of the charge density $\rho$ to a reference value: $\int_{\Omega}\left(\rho-\rho^{\text {ref }}\right)^{2}$ is shown.

The following observations can be made from both figures. As expected, the IGA with $C^{0}$ continuity is exactly equivalent to the FEM. The higher degree of continuity in IGA leads to an improved convergence with respect to $N_{\mathrm{nz}}$ and $N_{\text {dof }}$, and a worse convergence with respect to $N_{c}$.

The computations were executed on a 12 core computer (AMD Opteron ${ }^{\mathrm{TM}}$ Processor 4184, $800 \mathrm{MHz}, 128 \mathrm{~GB}$ RAM), with a BLAS level parallelization employed. The times, per DFT loop iteration, required for the matrix assembling, the evaluation of the Poisson problem boundary conditions (9) in all boundary quadrature points, the Poisson problem (12) solution, the Kohn-Sham eigenvalue problem (11) solution (min. and max. of all DFT loop iterations) and the average total time per DFT loop iteration for selected grid sizes are shown in Table 4, as well as the rank-m update part evaluation of $\boldsymbol{V}\left(\psi_{i}\right)$ that needs to be computed only once for each particular arrangement of atoms (iteration 1 of the DFT loop). The eigenvalue problem solution time was decreasing progressively as the self-consistent iteration converged (e.g. from 26 to 10 min for the FEM basis problem). About 10 DFT loop iterations were required to achieve the self-consistent solution. The largest computation used about $40 \mathrm{~GB}$ of RAM (the last row in Table 4).

The ratio of the eigenvalue problem solution time vs. the assembling time increased with the problem size, making the eigenvalue solver one of the bottlenecks of the computation for large problems, especially for bases with $C^{0}$ global continuity. For large $C^{2}$ globally continuous IGA basis, the assembling and rank-m update evaluation costs were significant, although the latter needed to be evaluated only in the first DFT loop iteration. We do not consider the very costly evaluation of the boundary conditions for the Poisson problem to be as problematic: this could be mitigated by using a better quadrature scheme, and a trivial parallelization (not done in the above computations). Via the code optimization, code parallelization, and mesh optimization, the performance can be significantly improved, which however was not our aim while making the test calculations for the purpose of this paper. We pursue the performance improvements in subsequent work. 

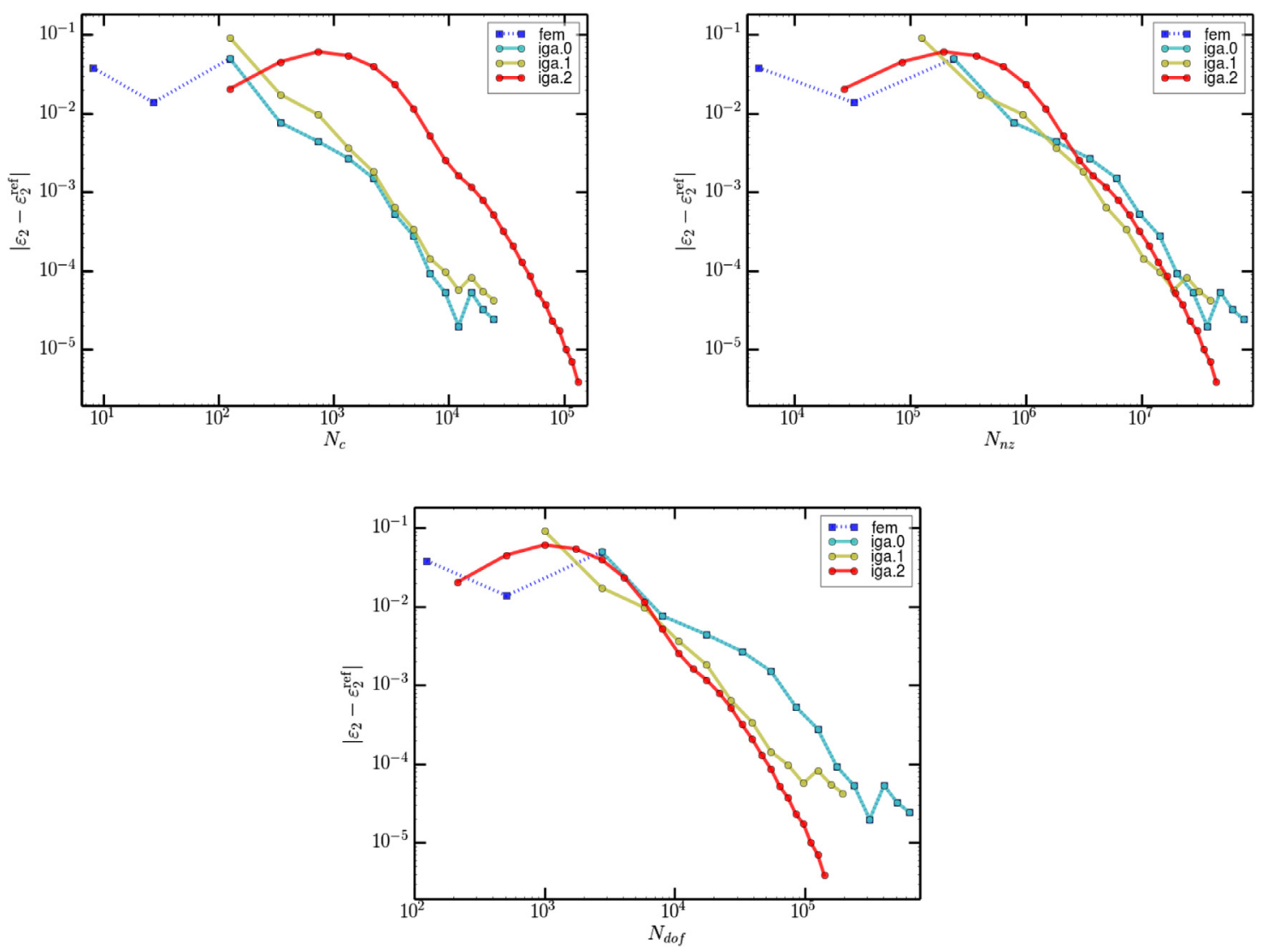

Fig. 8. Convergence with respect to the number of assembling cells $N_{c}$ (top-left), the number of non-zero entries in the matrices $N_{\mathrm{nz}}$ (top-right) and the total number of degrees of freedom $N_{\text {dof }}$ (bottom) of the second (triple) eigenvalue $\varepsilon_{2}$ : difference with respect to a reference value. The IGA labels indicate the global basis continuity. The tri-cubic FEM basis and degree three IGA (B-spline) basis were used.

\section{Table 4}

Typical times per DFT loop iteration for the largest FEM and IGA grid sizes. Elapsed times required for matrix assembling (Assem.), evaluation of Poisson problem boundary conditions in all boundary quadrature points (Poisson BC), the Poisson problem solution (Poisson solve), the Kohn-Sham eigenvalue problem solution: min. and max. of all DFT loop iterations (Kohn-Sham) and the total average time per DFT loop iteration (Total). The rank-m update part evaluation time of $\boldsymbol{V}\left(\psi_{i}\right)$ (rank-m eval.) is specific to iteration 1 only. The times are in minutes.

\begin{tabular}{llllllllll}
\hline Basis & Cont. & $N_{\text {dof }}$ & $N_{e}\left(N_{c}\right)$ & $\begin{array}{l}\text { Rank-m } \\
\text { eval. } \\
(\mathrm{min})\end{array}$ & $\begin{array}{lllll}\text { Assem. } \\
(\mathrm{min})\end{array}$ & $\begin{array}{l}\text { Poisson } \\
\mathrm{BC} \\
(\mathrm{min})\end{array}$ & $\begin{array}{l}\text { Poisson } \\
\text { solve } \\
(\mathrm{min})\end{array}$ & $\begin{array}{l}\text { Kohn-Sham } \\
(\mathrm{min}-\mathrm{max}) \\
(\mathrm{min})\end{array}$ & $\begin{array}{l}\text { Total } \\
(\text { avg. } \\
(\mathrm{min})\end{array}$ \\
\hline fem & 0 & 636,056 & $30(24389)$ & 0.5 & 2.3 & 3 & 2.7 & $10-26$ & 21 \\
iga & 0 & 636,056 & $30(24389)$ & 4.8 & 2.5 & 5 & 3.3 & $10-27$ & 24 \\
iga & 1 & 195,112 & $30(24389)$ & 4.8 & 2.6 & 5 & 1.7 & $5-13$ & 26 \\
iga & 2 & 27,000 & $30(24389)$ & 4.9 & 3 & 8 & 1.4 & $0.6-1.2$ & 14 \\
iga & 2 & 140,608 & $52(132651)$ & 27 & 17 & 84 & 8 & $8-22$ & 120 \\
\hline
\end{tabular}

\subsection{Results summary}

We were interested in the convergence with respect to the following three parameters: the number of assembling cells $N_{c}$ ( $\approx$ cost of quadrature and assembling; the assembling cells are elements for FEM, knot spans/Bézier elements for IGA), the number of non-zero entries in the matrices $N_{n z}(\approx$ cost of matrix-vector products) and the total number of degrees of freedom $N_{\text {dof }}$. The convergence of the Poisson equation solution to a manufactured analytic solution, the convergence of the two smallest eigenvalues of simple quantum mechanical systems and finally the convergence of the complete DFT loop were assessed.

As reported in Section 4.1, in all the tests, IGA with $C^{2}$ global continuity is the most efficient in terms of the convergence with respect to $N_{\text {dof }}$, i.e., the sizes of the vectors and matrices involved, which relate to the difficulty of solving the Poisson Eq. (8) or the eigenvalue problem (7). 

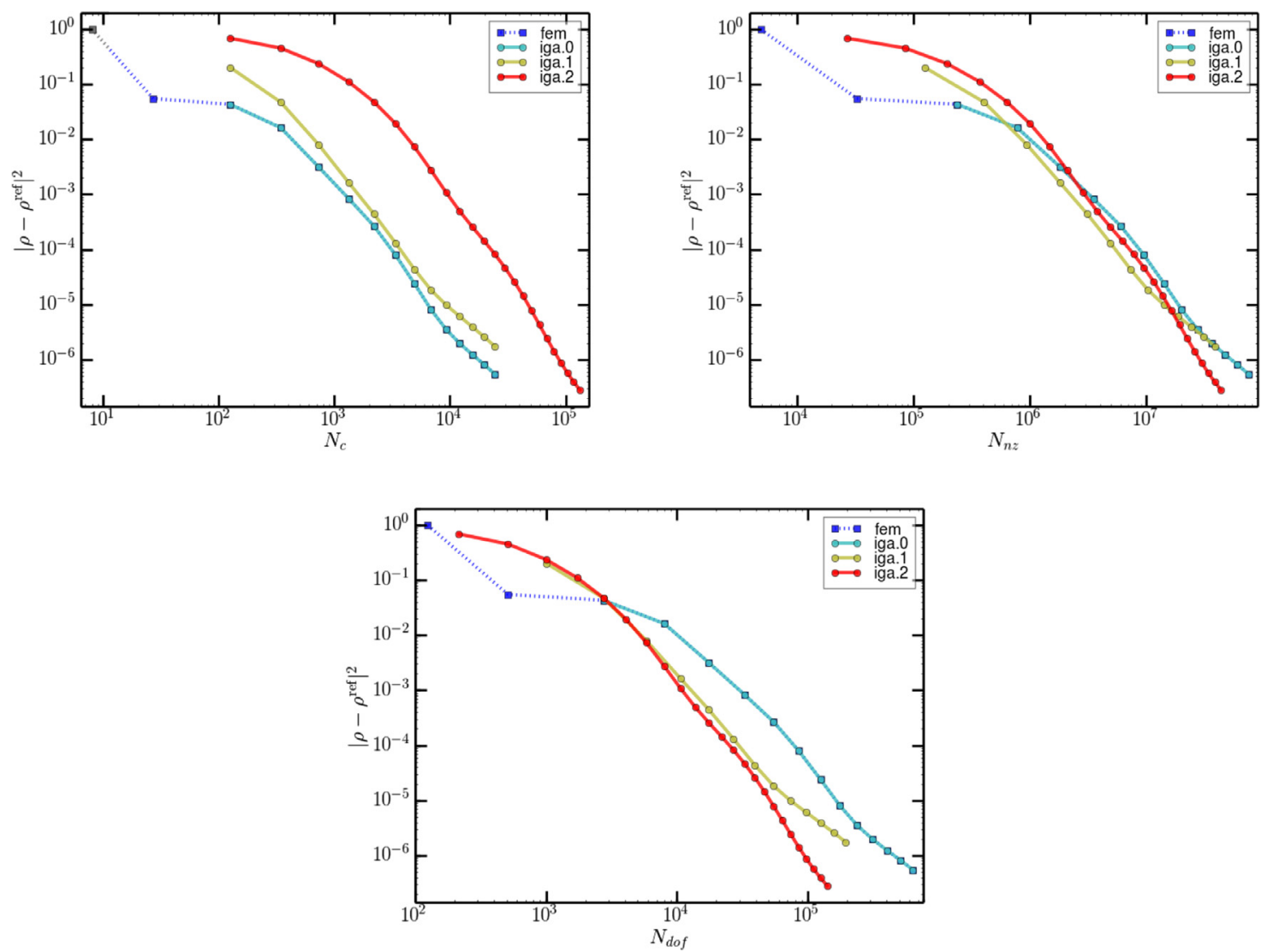

Fig. 9. Convergence with respect to the number of assembling cells $N_{c}$ (top-left), the number of non-zero entries in the matrices $N_{\mathrm{nz}}$ (top-right) and the total number of degrees of freedom $N_{\text {dof }}$ (bottom) of the charge density $\rho$ : difference with respect to a reference value. The IGA labels indicate the global basis continuity. The tri-cubic FEM basis and degree three IGA (B-spline) basis were used.

In the Poisson equation test problems, IGA with the increasing continuity performs progressively better than FEM with the same polynomial order when the convergence with respect to $N_{\mathrm{nz}}$ and $N_{\mathrm{dof}}$ is considered. On the other hand, the standard $C^{0}$ basis performs better when considering the convergence with respect to $N_{c}$. Our numerical results were consistent with the theoretical convergence results in [5].

Similar results were obtained for the eigenvalue problems in Section 4.2 originating from simple quantum mechanical systems. Again, IGA with the increasing continuity performs progressively better than FEM with the same polynomial order considering the convergence with respect to $N_{\mathrm{nz}}$ and $N_{\mathrm{dof}}$, and the standard $C^{0}$ basis performs better when considering the convergence with respect to $N_{c}$.

Considering the convergence of the eigenvalues $\varepsilon_{1}, \varepsilon_{2}$ and the charge density $\rho$ for the nitrogen atom benchmark computed by the complete DFT loop in Section 4.3, increasing the IGA basis continuity improves again the convergence with respect to $N_{\mathrm{nz}}$ and $N_{\text {dof. }}$. In the case of the convergence with respect to $N_{c}$, however, increasing the basis continuity (and thus decreasing $N_{\text {dof }}$ for the same number of elements) leads to a significantly worse convergence. This corresponds to a much higher numerical quadrature cost of the $C^{2}$ IGA basis when compared to a $C^{0}$ basis of the same accuracy, and negates the positive effect of the smaller number of DOFs on the eigenvalue problem solution time.

\section{Conclusion}

We compared numerical convergence properties of FEM and IGA based on the Bézier extraction using problems originating from various stages of our electronic structure calculation algorithm [13,51], based on the density functional theory, the environment-reflecting pseudopotentials and a weak solution of the Kohn-Sham equations. Our computer implementation built upon the open source package SfePy supports computations both with the FE basis and the NURBS or B-splines basis of IGA. The latter allows a high global continuity in approximation of unknown fields, so convergence properties of B-spline bases with global continuities up to $C^{2}$ were examined, because having a globally $C^{2}$ continuous approximation is crucial for the efficient calculation of derivatives of the total energy with respect to atomic positions etc., as given by the Hellmann-Feynman theorem [13]. 
Overall, the results summarized in Section 4.4 support our choice of IGA as a viable alternative to FEM in electronic structure calculations. To alleviate the numerical quadrature cost, reduced quadrature rules have been proposed for the context of the Bézier extraction [44], which we plan to assess in future.

\section{Acknowledgments}

The work was supported by the Czech Science Foundation, grant projects GAP 108/11/0853 (initial part) and GA17$12925 \mathrm{~S}$ (very recent calculations). The second author acknowledges the support by CEDAMNF project, reg. no. CZ. 02.1.01/0.0/0.0/15_003/0000358, co-funded by the ERDF as part of the Ministry of Education, Youth and Sports OP RDE programme. The work of R. Kolman and J. Plešek was supported by the Centre of Excellence for Nonlinear Dynamic Behaviour of Advanced Materials in Engineering CZ.02.1.01/0.0/0.0/15_003/0000493 (Excellent Research Teams) in the framework of Operational Programme Research, Development and Education, within institutional support RVO:61388998.

\section{References}

[1] N.W. Ashcroft, N.D. Mermin, Solid State Physics, Holt, Rinehart and Winston, New York, 1976

[2] S. Balay, S. Abhyankar, M.F. Adams, J. Brown, P. Brune, K. Buschelman, L. Dalcin, V. Eijkhout, W.D. Gropp, D. Kaushik, M.G. Knepley, L.C. McInnes, K. Rupp, B.F. Smith, S. Zampini, H. Zhang, H. Zhang, PETSc Users Manual, Technical Report ANL-95/11 - Revision 3.7, Argonne National Laboratory, 2016. URL http://www.mcs.anl.gov/petsc.

[3] B. Bastl, M. Brandner, J. Egermaier, K. Michálková, E. Turnerová, Isogeometric analysis for turbulent flow, Math. Comput. Simul. (2016), doi:10.1016/j. matcom.2016.05.010.

[4] Y. Bazilevs, V.M. Calo, J.A. Cottrell, J.A. Evans, T.J.R. Hughes, S. Lipton, M.A. Scott, T.W. Sederberg, Isogeometric analysis using T-splines, Comput. Methods Appl. Mech. Eng. 199 (2010) 229-263.

[5] Y. Bazilevs, L.B.A. da Veiga, J.A. Cottrell, T.J.R. Hughes, G. Sangalli, Isogeometric analysis: approximation, stability and error estimates for h-refined meshes, Math. Models Methods Appl. Sci. 16 (2011) 1031-1090.

[6] L. Beiráo da Veiga, A. Buffa, J. Rivas, G. Sangalli, Some estimates for $h-p-k$-refinement in isogeometric analysis, Numerische Mathematik 118 (2) (2011) 271-305.

[7] F. Bloch, Über die quantenmechanik der elektronen in kristallgittern, Z. Phys. 52 (1928) 555-600, doi:10.1007/BF01339455.

[8] M.J. Borden, M.A. Scott, J.A. Evans, T.J.R. Hughes, Isogeometric finite element data structures based on Bezier extraction of NURBS, Int. J. Numer. Methods Eng. 87 (2011) 15-47, doi:10.1002/nme.2968.

[9] P. Bornemann, F. Cirak, A subdivision-based implementation of the hierarchical b-spline finite element method, Comput. Methods Appl. Mech. Eng. 253 (2013) 584-598. http://dx.doi.org/10.1016/j.cma.2012.06.023.

[10] A. Buffa, G. Sangalli, C. Schwab, Exponential convergence of the hp version of isogeometric analysis in 1d, in: Spectral and High Order Methods for Partial Differential Equations - ICOSAHOM 2012, in: Lecture Notes in Computational Science and Engineering, vol. 95, Springer International Publishing, 2014, pp. 191-203.

[11] R. Cimrman, Enhancing SfePy with isogeometric analysis, in: P. de Buyl, N. Varoquaux (Eds.), Proceedings of the 7th European Conference on Python in Science (EuroSciPy 2014), 2014a, pp. 65-72. URL http://arxiv.org/abs/1412.6407.

[12] R. Cimrman, SfePy - write your own FE application, in: P. de Buyl, N. Varoquaux (Eds.), Proceedings of the 6th European Conference on Python in Science (EuroSciPy 2013), 2014b, pp. 65-70. URL http://arxiv.org/abs/1404.6391.

[13] R. Cimrman, M. Novák, R. Kolman, M. Tuma, J. Vackář, Isogeometric analysis in electronic structure calculations, Math. Comput. Simul. (2016), doi:10. 1016/j.matcom.2016.05.011.

[14] J.A. Cottrell, T.J.R. Hughes, Y. Bazilevs, Isogeometric Analysis: Toward Integration of CAD and FEA, John Wiley \& Sons, 2009.

[15] J.A. Cottrell, T.J.R. Hughes, A. Reali, Studies of refinement and continuity in isogeometric structural analysis, Comput. Methods Appl. Mech. Eng. 196 (2007) 4160-4183.

[16] J.A. Cottrell, A. Reali, Y. Bazilevs, T.J.R. Hughes, Isogeometric analysis of structural vibrations, Comput. Methods Appl. Mech. Eng. 195 (41-43) (2006) 5257-5296.

[17] L.D. Dalcin, R.R. Paz, P.A. Kler, A. Cosimo, Parallel distributed computing using Python, Adv. Water Resourc. 34 (9) (2011) 1124-1139. http://dx.doi.org/ 10.1016/j.advwatres.2011.04.013. New Computational Methods and Software Tools

[18] T.A. Davis, Algorithm 832: UMFPACK v4.3-an unsymmetric-pattern multifrontal method, ACM Trans. Math. Software 30 (2) (2004) 196-199, doi:10. $1145 / 992200.992206$.

[19] D. Davydov, T. Gerasimov, J.-P. Pelteret, P. Steinmann, On the h-adaptive PUM and the hp-adaptive FEM approaches applied to PDEs in quantum mechanics, 2017. ArXiv:1612.02305 [physics.comp-ph].

[20] D. Davydov, T.D. Young, P. Steinmann, On the adaptive finite element analysis of the Kohn-Sham equations: methods, algorithms, and implementation, Int. J. Numer. Methods Eng. 106 (11) (2015) 863-888, doi:10.1002/nme.5140.

[21] R.M. Dreizler, E.K.U. Gross, Density Functional Theory, Springer-Verlag, 1990.

[22] M.J. Frisch et al., Gaussian 09, Revision E.01. Gaussian Inc. Wallingford CT, 2009

[23] V. Gavini, K. Bhattacharya, M. Ortiz, Quasi-continuum orbital-free density-functional theory: A route to multi-million atom non-periodic DFT calculation, J. Mech. Phys. Solids 55 (4) (2007) 697-718, doi:10.1016/j.jmps.2007.01.012.

[24] R. Geus, D. Wheeler, D. Orban, Pysparse documentation. URL http://pysparse.sourceforge.net.

[25] T.J.R. Hughes, The Finite Element Method: Linear Static and Dynamic Finite Element Analysis, Dover Publications, 2000.

[26] T.J.R. Hughes, J.A. Evans, A. Reali, Finite element and NURBS approximations of eigenvalue, boundary-value, and initial-value problems, Comput. Methods Appl. Mech. Eng. 272 (2014) 290-320.

[27] T.J.R. Hughes, A. Reali, G. Sangalli, Duality and unified analysis of discrete approximations in structural dynamics and wave propagation: comparison of p-method finite elements with k-method NURBS, Comput. Methods Appl. Mech. Eng. 197 (49-50) (2008) 4104-4124.

[28] J. Ihm, A. Zunger, M.L. Cohen, Momentum-space formalism for the total energy of solids, J. Phys. C: Solid State Phys. 12 (1979) $4409-4422$.

[29] C. Kittel, Introduction to Solid State Physics, Wiley, New York, 1996.

[30] W. Kohn, L.J. Sham, Self-consistent equations including exchange and correlation effects, Phys. Rev. 140 (4A) (1965) A1133-A1138, doi:10.1103/PhysRev. 140.A1133.

[31] R. Kolman, J. Plešek, M. Okrouhlík, Complex wavenumber Fourier analysis of the B-spline based finite element method, Wave Motion 51 (2013) 348-359.

[32] R. Kolman, S.V. Sorokin, B. Bastl, J. Kopačka, J. Plešek, Isogeometric analysis of free vibration of simple shaped elastic samples, J. Acoust. Soc. Am. 137 (4) (2015) 2089-2100

[33] M. Kästner, P. Metsch, R. de Borst, Isogeometric analysis of the Cahn-Hilliard equation - a convergence study, J. Comput. Phys. 305 (C) (2016) 360-371, doi:10.1016/j.jcp.2015.10.047.

[34] R.M. Martin, Electronic Structure: Basic Theory and Practical Methods, Cambridge University Press, 2005 
[35] A. Masud, R. Kannan, B-splines and NURBS based finite element methods for Kohn-Sham equations, Comput. Methods Appl. Mech. Eng. 241-244 (2012) 112-127, doi:10.1016/j.cma.2012.04.016.

[36] P. Motamarri, V. Gavini, Subquadratic-scaling subspace projection method for large-scale Kohn-Sham density functional theory calculations using spectral finite-element discretization, Phys. Rev. B 90 (2014) 115127, doi:10.1103/PhysRevB.90.115127.

[37] P. Motamarri, M.R. Nowak, K. Leiter, J. Knap, V. Gavini, Higher-order adaptive finite-element methods for Kohn-Sham density functional theory, J. Comput. Phys. 253 (2013) 308-343, doi:10.1016/j.jcp.2013.06.042.

[38] R.G. Parr, Y. Weitao, Density-Functional Theory of Atoms and Molecules, Oxford University Press, USA, 1994.

[39] J. Pask, N. Sukumar, Partition of unity finite element method for quantum mechanical materials calculations, Extreme Mech. Lett. 11 (2017) 8-17. http://dx.doi.org/10.1016/j.eml.2016.11.003.

[40] J.E. Pask, B.M. Klein, C.Y. Fong, P.A. Sterne, Real-space local polynomial basis for solid-state electronic-structure calculations: a finite-element approach, Phys. Rev. B 59 (1999) 12352-12358, doi:10.1103/PhysRevB.59.12352.

[41] W.E. Pickett, Pseudopotential methods in condensed matter applications, Comput. Phys. Rep. 9 (1989) 115-198.

[42] L. Piegl, W. Tiller, The NURBS Book, second ed., Springer-Verlag, 1995-1997.

[43] V. Schauer, C. Linder, The reduced basis method in all-electron calculations with finite elements, Adv. Comput. Math. 41 (5) (2015) 1035-1047, doi:10. 1007/s10444-014-9374-z.

[44] D. Schillinger, S.J. Hossain, T.J.R. Hughes, Reduced Bézier element quadrature rules for quadratic and cubic splines in isogeometric analysis, Comput. Methods Appl. Mech. Eng. 277 (2014) 1-45, doi:10.1016/j.cma.2014.04.008.

[45] G. Strang, G. Fix, An Analysis of the Finite Element Method, Wellesley-Cambridge Press, Wellesley, 2008, p. 414.

[46] N. Sukumar, J.E. Pask, Classical and enriched finite element formulations for Bloch-periodic boundary conditions, Int. J. Numer. Methods Eng. 77 (8) (2009) 1121-1138, doi:10.1002/nme.2457.

[47] P. Suryanarayana, K. Bhattacharya, M. Ortiz, A mesh-free convex approximation scheme for Kohn-Sham density functional theory, J. Comput. Phys. 230 (13) (2011) 5226-5238, doi:10.1016/j.jcp.2011.03.018.

[48] P. Suryanarayana, K. Bhattacharya, M. Ortiz, Coarse-graining Kohn-Sham density functional theory, J. Mech. Phys. Solids 61 (1) (2013) 38-60, doi:10. 1016/j.jmps.2012.09.002.

[49] P. Suryanarayana, V. Gavini, T. Blesgen, K. Bhattacharya, M. Ortiz, Non-periodic finite-element formulation of Kohn-Sham density functional theory, J. Mech. Phys. Solids 58 (2) (2010) 256-280, doi:10.1016/j.jmps.2009.10.002.

[50] J. Vackáŕ, A. Šimuunek, Adaptability and accuracy of all-electron pseudopotentials, Phys. Rev. B 67 (2003). 125113

[51] J. Vackář, O. Čertík, R. Cimrman, M. Novák, O. Šipr, J. Plešek, Advances in the Theory of Quantum Systems in Chemistry and Physics, Prog. Theoretical Chem. and Phys., vol. 22, Springer, 2011, pp. 199-217. 